\title{
What Can (and Can't) we Do with Sparse Polynomials?
}

\author{
Daniel S. Roche \\ United States Naval Academy \\ Annapolis, Maryland, U.S.A. \\ roche@usna.edu
}

\begin{abstract}
Simply put, a sparse polynomial is one whose zero coefficients are not explicitly stored. Such objects are ubiquitous in exact computing, and so naturally we would like to have efficient algorithms to handle them. However, with this compact storage comes new algorithmic challenges, as fast algorithms for dense polynomials may no longer be efficient. In this tutorial we examine the state of the art for sparse polynomial algorithms in three areas: arithmetic, interpolation, and factorization. The aim is to highlight recent progress both in theory and in practice, as well as opportunities for future work.
\end{abstract}

\section{KEYWORDS}

sparse polynomial, interpolation, arithmetic, factorization

\section{ACM Reference Format:}

Daniel S. Roche. 2018. What Can (and Can't) we Do with Sparse Polynomials?. In ISSAC '18: 2018 ACM International Symposium on Symbolic and Algebraic Computation, fuly 16-19, 2018, New York, NY, USA. ACM, New York, NY, USA, 6 pages. https://doi.org/10.1145/3208976.3209027

\section{SPARSE POLYNOMIALS}

Sparse polynomials are found in the core of nearly every computer algebra system or library, and polynomials with many zero coefficients frequently occur in practical settings.

Mathematically, the dividing line between a sparse and dense polynomial is not well-defined. From a computer science standpoint, there is a clear distinction, depending on the representation of that polynomial in memory: A dense representation stores zero coefficients explicitly and exponents implicitly, whereas a sparse representation does not store zero coefficients at all, but stores exponents explicitly.

There are many variants of sparse representations [25]. This tutorial considers algorithms for the most compact representation, the so-called distributed sparse storage [72]. Let $f \in \mathrm{R}\left[x_{1}, \ldots, x_{n}\right]$ be an $n$-variate polynomial with coefficients in a ring R. The representation of $f$ is by a list of $t$ nonzero terms

$$
\left(c_{1}, e_{1,1}, \ldots, e_{1, n}\right),\left(c_{2}, e_{2,1}, \ldots, e_{2, n}\right), \ldots,\left(c_{t}, e_{t, 1}, \ldots, e_{t, n}\right)
$$

such that

$$
f=c_{1} x_{1}^{e_{1,1}} \cdots x_{n}^{e_{1, n}}+c_{2} x_{1}^{e_{2,1}} \cdots x_{n}^{e_{2, n}}+\cdots+c_{t} x_{1}^{e_{t, 1}} \cdots x_{n}^{e_{t, n}}
$$

with each coefficient $c_{i} \in \mathrm{R}$ nonzero and all exponent tuples $\left(e_{i, 1}, \ldots, e_{i, n}\right) \in \mathbb{N}^{n}$ distinct. We also assume that the terms are sorted according to their exponents in some consistent way.

This paper is authored by an employee(s) of the United States Government and is in the public domain. Non-exclusive copying or redistribution is allowed, provided that the article citation is given and the authors and agency are clearly identified as its source.

ISSAC '18, fuly 16-19, 2018, New York, NY, USA

2018. ACM ISBN 978-1-4503-5550-6/18/07.

https://doi.org/10.1145/3208976.3209027
This sparse representation matches the one used by default for multivariate polynomials in modern computer algebra systems and libraries such as Magma [83], Maple [73], Mathematica, Sage [84], and Singular [81].

\subsection{Sparse polynomial algorithm complexity}

Sparse polynomials in the distributed representation are also called lacunary or supersparse [55] in the literature to emphasize that, in this representation, the degree of a polynomial could be exponentially larger than its bit-length. This exposes the essential difficulty of computing with sparse polynomials, in that efficient algorithms for dense polynomials may cost exponential-time in the sparse setting.

Specifically, when analyzing algorithms for dense polynomials, the most important measure is the degree bound $D \in \mathbb{N}$ such that $\operatorname{deg} f<D$. The size of the dense representation of a univariate polynomial is $D$ ring elements, and many operations can be performed in $D^{O(1)}$ ring operations, or even $D(\log D)^{O(1)}$.

In the sparse representation, we need to also consider the number of nonzero terms $t$, and the bit-length of the exponents. For a multivariate (sparse) polynomial, the representation size is $O(t)$ ring elements plus $O(n t \log D)$ bits, where $n$ is the number of variables and $D$ is now an upper bound on the maximum degree. The goal, then, is to develop new sparse polynomial algorithms which minimize the cost in terms of $n, t$, and $\log D$.

The coefficient ring $\mathrm{R}$ makes a difference for some algorithms. In the general setting we let $\mathrm{R}$ be an arbitrary integral domain, and count ring operations. Another important setting is when $R=\mathbb{Z}$, the ring of integers, in which case we also account for the size of coefficients. Write $\mathrm{H}(f)$ for the height of a polynomial, which is the maximum magnitude $\max _{i}\left|c_{i}\right|$ of its coefficients; then a fast algorithm on $f$ should have a small running time in terms of $\log \mathrm{H}(f)$.

For simplicity of presentation, and because the algorithmic work with sparse polynomials is still at a much more coarse level than, say, that of integers and dense polynomials, we frequently use the soft-oh notation $\widetilde{O}(\gamma):=O\left(\gamma \cdot(\log \gamma)^{O(1)}\right)$, where $\gamma$ is some running-time function.

\subsection{Overview}

The basic challenge of sparse polynomial algorithms is to match the complexity of dense polynomial algorithms for the same task. In some cases, interestingly, this is (provably) not possible - for a few problems, even a polynomial-time algorithm in the sparse representation size would imply that $\mathbf{P}=\mathrm{NP}$.

Where algorithms are possible, one interesting feature is that they must usually take into account not only the coefficient arithmetic over $\mathrm{R}$, but also the exponent arithmetic over $\mathbb{Z}$. In fact, the latter frequently poses the most difficulty in the design of efficient algorithms. 
This tutorial aims to outline the state of sparse polynomial algorithms, dividing roughly into the three areas of arithmetic, interpolation, and factorization. We highlight where essentially-optimal algorithms are already known, where they are known not to exist, and the numerous cases of open problems in between.

\section{ARITHMETIC}

Polynomials stored in the dense representation can be added and subtracted in linear-time. Dense polynomial multiplication costs $O\left(D^{2}\right)$ ring operations using the classical algorithm, but considerable research effort has gone to reducing this complexity, which we now denote as simply $M(D)$. The most general result of [16] gives $\mathrm{M}(D) \in O(D \log D \log \log n)$, and more recent work [27, 44] reduces this even further for most commonly-used rings.

Many other polynomial computations can be reduced to multiplication. In particular, Euclidean division with remainder costs $O(M(D))$, and (extended) gcd, multi-point evaluation, interpolation, Chinese remaindering, and rational reconstruction all cost $O(M(D) \log D)$ ring operations $[14, \S 2-6][32, \S 8-11]$. Note that all these operations take quasi-linear time in the input size $\widetilde{O}(D)$.

As discussed previously, these algorithms are not polynomialtime in the size of the sparse representation.

\subsection{Addition and subtraction}

Adding or subtracting sparse polynomials is a matter of combining like terms, which amounts to a merge operation on the two lists of nonzero terms. From our assumption that terms are stored in sorted order, this costs $O(t \log D)$ bit operations and $O(t)$ ring additions, where $t$ is the number of terms in the two input polynomials. This matches the size of the input and is therefore optimal.

Notice, however, that the size of the output can grow much more quickly than with dense polynomials. When adding two dense polynomials with degrees less than $D$, the cost is $O(D)$ and the output size is also $D$; the size does not increase. But when adding two $t$-sparse polynomials, the number of nonzero terms in the output may double to $2 t$. Hence repeated addition of sparse polynomials (of which multiplication is a special case) requires more care.

\subsection{Multiplication}

A significant difference from case of dense polynomial multiplication is that the output size grows quadratically: the product of multiplying two $t$-sparse polynomials may have as many as $t^{2}$ nonzero terms.

In terms of input size, therefore, the best that one can hope for is $O\left(t^{2}\right)$ ring operations and $O\left(t^{2} \log D\right)$ bit complexity. This is nearly achieved by the classical algorithm of repeated monomial multiplications and additions, which has bit complexity $O\left(t^{2} \log D \log t\right)$ if the additions (merges) are done in a balanced way [23, 24, 52].

In practice, the size of intermediate results, and in particular the time to handle memory allocation and de-allocation, can dominate the complexity of multiplication. At least one specialized data structure was designed for this purpose [85], and [52] suggested a very simple idea of using a heap to simultaneously store and sort un-merged terms in the product.

Decades later, Monagan and Pearce $[69,70,73,74]$ rediscovered the heaps idea and developed extremely efficient implementations and a careful analysis. They point out that, while the asymptotic runtime is the same, the space for intermediate results is only $O(t)$, no matter how many terms are in the output. This has tremendous practical performance benefits, and it seems that many computer algebra systems now use this approach in at least some cases. In fact, there seems to be considerable interest in the fastest practical speeds for sparse polynomial multiplication on a variety of hardware platforms including parallel computing [10, 29, 30, 70, 77].

Still, this is unsatisfying from an algorithmic perspective since the number of ring and bit-operations is still quadratic in every case, meaning that even the heap-based algorithms will have a hard cut-off with the speed of dense multiplication as polynomials start to fill-in with nonzero coefficients.

Another approach is to consider the size of the output as a parameter in the complexity. In the worst case, this can be quadratic in the input size, but in cases where the output is smaller, we can hope for faster computation. Furthermore, considering the output size allows for a smooth transition to dense algorithms, where the output is guaranteed to have at most $2 D$ nonzero terms.

Open Problem 1. Develop an algorithm to multiply two sparse polynomials $f, g \in \mathrm{R}[x]$ using $\widetilde{O}(t \log D)$ ring and bit operations, where $t$ is the number of terms in $f, g$, and $f g$, and $D$ is an upper bound on their degrees.

Considerable progress has been made toward this open problem, and it seems now nearly within reach. Some authors have looked at special cases, when the support of nonzero coefficients has a certain structure, to reduce to dense multiplication and achieve the desired complexity in those cases $[45,79,80]$.

A more general solution approach is to use sparse interpolation algorithms, which we examine in further detail in the next section. First, [46] showed that the open problem is essentially solved when the support of the product is already known. More recently, [2, 5] solved Open Problem 1 under two conditions:

- The ring $\mathrm{R}$ is either the integers $\mathbb{Z}$, or some other ring such as $\mathbb{F}_{p}$ that can be reduced to that case with no loss of efficiency.

- The so-called structural support matches the size of the actual support. Which is to say (roughly) that there are not too many cancellations of coefficients in the product.

Removing the second condition seems to be the main remaining hurdle in solving Open Problem 1.

\subsection{Division}

When dividing sparse polynomials, it is imperative to consider the output size: for example, the exact division of two 2-term polynomials $x^{D}-1$ by $x-1$ produces a quotient with $D$ nonzero terms.

Fortunately, the heaps idea which works well in practice for sparse multiplication has also been adapted to sparse division, and this method easily yields the remainder as well as the quotient $[31,71]$. As before, this approach uses $O\left(t^{2}\right)$ ring operations, leaving us with another challenge:

Open Problem 2. Given two sparse polynomials $f, g \in \mathrm{R}[x]$, develop an algorithm to compute the quotient and remainder $q, g \in$ $\mathrm{R}[x]$ such that $f=q g+r$, using $\widetilde{O}(t \log D)$ ring and bit operations, where $t$ is the number of terms of $f, g, q$, and $g$, and $\operatorname{deg} f<D$. 
Note that an efficient solution to Open Problem 2 is already available when $\operatorname{deg} g$ is small, i.e., when $f$ is sparse but $g$ is dense. The algorithm in that case amounts to computing $x^{e_{i}} \bmod g$ for all exponents $e_{i}$ that appear in the support of $f$, via repeated squaring, then multiplying by the coefficients $c_{i}$ and summing.

In the more difficult case that $g$ is also sparse, [22, §III] has a nice discussion of the problem. In particular, they point out that a seemingly-easier decision problem is still open:

Open Problem 3. Given two sparse polynomials $f, g \in \mathrm{R}[x]$, develop an algorithm which determines whether $g$ divides $f$ exactly, using $\widetilde{O}(t \log D)$ ring and bit operations.

Again, a solution is known only when $g$ is dense.

\section{INTERPOLATION}

Polynomial interpolation is a problem of model fitting: given some measurements, find a (sparse) polynomial which (best) fits the data.

In the case of dense polynomials, this is a classical problem. Exact interpolation from an arbitrary set of points can be accomplished in $\widetilde{O}(D)$ ring operations. Even if the data is noisy or has outliers, classical numerical methods can recover the best polynomial fit (see, e.g., $[82, \S 4,13]$ ).

The challenge of sparse polynomial interpolation is to fit a $t$ sparse polynomial to some small number of evaluation points $m \ll$ $D$. Even the decidability of this question is non-trivial and depends on the choice of ring and evaluation points [12]. In fact, all efficient solutions require a stronger model that allows the algorithm to sample the unknown function at arbitrary points.

Definition 4. A black box for an unknown $n$-variate polynomial $f \in \mathrm{R}\left[x_{1}, \ldots, x_{n}\right]$ is a function which accepts any $n$-tuple $\left(\theta_{1}, \ldots, \theta_{n}\right) \in \mathrm{R}^{n}$ and produces the value $f\left(\theta_{1}, \ldots, \theta_{n}\right) \in \mathrm{R}$.

In this context, a sparse interpolation algorithm takes a black box for an unknown $f$ as well as upper bounds $D, T$ on its degree and number of nonzero terms, respectively. An efficient algorithm should minimize the number of evaluations, plus required ring and bit-operations, in terms of $T$ and $\log D$.

Open Problem 5. For any ring R, given a black box for $f \in$ $\mathrm{R}\left[x_{1}, \ldots, x_{n}\right]$ and bounds $T, D$ on the number of nonzero terms and maximum degree of $f$, determine the nonzero coefficients and corresponding exponents of $f$ using $\widetilde{O}(T \log D)$ ring operations, bit operations, and black box evaluations.

The methods for sparse interpolation have very strong connections to other techniques in coding theory and signal processing. In the first case, the support of the unknown sparse polynomial $f$ corresponds to the error locations in Reed-Solomon decoding; the decoding algorithm of Blahut [11] can be seen as a sparse interpolation algorithm similar to Prony's method below [18].

In another viewpoint, the evaluations of a sparse polynomial at integer powers of some complex root of unity have a 1-1 correspondence with evaluations of a sum of exponentials at integer points: signal frequencies correspond to polynomial exponents and amplitudes correspond to polynomial coefficients. The recovery techniques come from multi-exponential analysis, the theory of Padé approximants, and tensor decomposition; see [21, 75] and references therein.
In the context of computer algebra, algorithms for sparse polynomial interpolation go back to the work of Zippel [86, 87], who developed a randomized algorithm which recovers a sparse polynomial in recursively, variable-by variable. However, the reliance on dense univariate interpolation makes it unsuitable for the setting of this tutorial.

For exact (super)sparse polynomial interpolation, we first consider the easier case of univariate polynomials where $n=1$. There are are essentially two classes of algorithms here, which we discuss separately. Then we see how to reduce the multivariate case to the univariate one without changing the sparsity.

\subsection{Prony's method}

The classic numerical technique of Prony [78] from the 18th century was rediscovered by Ben-Or and Tiwari and adapted to the context of computer algebra nearly 200 years later [9]. The key idea is that any sequence of evaluations at consecutive values in geometric progression $\left(f\left(\omega^{i}\right)\right)_{i \geq 0}$ form a linearly-recurrent sequence with degree $t$, where $t$ is the actual number of nonzero terms in $f$. Furthermore, the minimum polynomial of the linear recurrence is a product of linear factors, and its roots are exactly of the form $\omega^{e_{i}}$, where $e_{i}$ is the exponent of a nonzero term in $f$.

Given a black box for unknown univariate sparse polynomial $f \in \mathrm{R}[z]$, plus degree and sparsity bounds $D$ and $T$, the algorithm takes the following steps:

(1) Find a suitable element $\omega \in \mathrm{R}$ with multiplicative order at least $D$.

(2) Evaluate $f(1), f(\omega), f\left(\omega^{2}\right), \ldots, f\left(\omega^{2 T-1}\right)$.

(3) Use the Berlekamp-Massey algorithm (or a Toeplitz solver) to compute the minimum polynomial of the linear recurrence $\Lambda(z) \in \mathrm{R}[z]$.

(4) Find the roots $\omega^{e_{i}}$ of $\Lambda$.

(5) Compute the discrete logarithms of the roots to base $\omega$ to discover the exponents $e_{1}, \ldots, e_{t}$.

(6) Solve a transposed Vandermonde system from the first $t$ evaluations to recover the nonzero coefficients $c_{1}, \ldots, c_{t}$.

The main benefit of this procedure is that it computes the minimal number of evaluations $2 T$ in Step 2. Steps 3 and 6 involve well-known techniques from structured linear algebra and can be solved efficiently using $\widetilde{O}(t)$ ring operations $[13,58]$.

However, the other steps depend heavily on the coefficient ring R. In particular, the algorithm must find a high-order element $\omega \in \mathrm{R}$, perform root-finding of a degree- $t$ polynomial, and then perform $t$ discrete logarithms to the base $\omega$.

When $\mathrm{R}=\mathbb{Z}$, these steps can be performed reasonably efficiently by working modulo $p$ for a very carefully-chosen prime $p$. The first modular version of this approach [60] was not polynomial-time in the discrete logarithm step, but an idea from $[53,61]$ proposes choosing a prime $p$ with $p \in O(D)$ such that $(p-1)$ is divisible by a large power of 2 . This divisor means that $\mathbb{F}_{p}^{*}$ has a large subgroup with smooth order, facilitating fast discrete logarithms in only $\widetilde{O}\left(\log ^{2} D\right)$ field operations each. Because of the size of the prime, the resulting algorithm has total cost of $\widetilde{O}\left(T \log ^{3} D+T \log \mathrm{H}(f)\right)$ bit operations. This almost solves Open Problem 5, except it is cubic in the size of the exponents $\log D$. 
Many important improvements have been made to this algorithm since it was developed in the 1990s. Early-termination techniques allow for only $O(t)$ evaluations instead of $O(T)$, where $t \leq T$ is the true sparsity of $f$ [57]. The root-finding step was found to be the bottleneck in a practical implementation by [47]; a better rootfinding algorithm in this case was developed [40] to improve the situation.

[51] adapt the algorithm to the case of finite fields with a parallel algorithm that has better complexity in terms of $\log D$ but becomes quadratic in the sparsity $t$; they also implemented their algorithm and performed some experiments. Earlier modular, parallel algorithms were also developed by $[42,48]$. A more straightforward parallel algorithm over $\mathbb{Z}$ was developed and experimentally evaluated by [65].

\subsection{Homomorphic imaging}

As we have seen, the Prony approach to sparse interpolation does not perform well over arbitrary finite fields due to the cost of discrete logarithm computations, which in general cannot be performed in polynomial-time.

A radically different method was first proposed by [28], based on some earlier ideas of [41]. This does not directly use the black box model defined earlier, but instead assumes a more generous model that can be stated as follows:

Definition 6. A modular black box for an unknown polynomial $f \in \mathrm{R}[x]$ is a function which accepts any pair of dense polynomials $g, h \in \mathrm{F}[x]$ with $\operatorname{deg} h<\operatorname{deg} g$ and produces the value $f(h) \bmod g$.

If $g=x$ and $h=\theta$, this corresponds to the normal black-box evaluation $f(\theta)$. But when $\operatorname{deg} g>1$, the setting is more general. It makes sense when interpolating a straight-line program or algebraic circuit for $f$, where each step of the evaluation can be performed modulo $g$. We must be careful with the cost model also, because for example if $\operatorname{deg} g>\operatorname{deg} f$, the problem is trivially solved with a single evaluation. To accommodate this, we say that each such evaluation costs $\widetilde{O}(\operatorname{deg} g)$ ring operations.

The first algorithm in this model by [28] was deterministic and works over any ring $\mathrm{R}$, but with a high complexity of $\widetilde{O}\left(T^{4} \log ^{2} D\right)$. A series of later improvements [6-8, 37, 49] has improved this to $\widetilde{O}\left(T \log ^{3} D\right)$ ring operations, largely by introducing numerous randomizations. Note that this is a similar cost to the best-known variants of Prony's method over $\mathbb{Z}$, but it still has the comparative shortcoming of requiring more expensive evaluations.

\subsection{Kronecker substitution}

Any $n$-variate polynomial $f$ with maximum degree less than $D$ is in one-to-one correspondence with a univariate polynomial $g$ with degree less than $D^{n}$, according to a map introduced by Kronecker [66]. The forward map can be written as an evaluation of $f$ at powers of a single variable $z$ :

$$
g(z):=f\left(z, z^{D}, \ldots, z^{D^{n-1}}\right) .
$$

The reverse map simply involves converting each integer exponent of $g$ into an $n$-tuple of exponents of $f$ via a $D$-adic expansion of the univariate exponent.
Because the forward map is simply an evaluation, this means that a multivariate $f$ can be found via univariate supersparse interpolation of a single polynomial with degree less than $D^{n}$ and the same number of nonzero terms. Supersparse algorithms have complexity in terms of the bit-length of the exponents, so the resulting complexity should be polynomial in $T$ and $n \log D$, as desired.

Even so, the exponential increase in degree is to be avoided, since the cost of both approaches above is at least quadratic in $\log D$. A compromise approach was presented by [4], who showed how to use a randomization to reduce the multivariate polynomial to a set of $O(n)$ univariate polynomials, each of degree only $O(D T)$. When combined with the univariate supersparse interpolation algorithms above, this results in a better complexity in terms of $n$.

\subsection{Problem variations and extensions}

Numerous authors have focused on solving different useful variants of the sparse interpolation problem rather than improving the asymptotic complexity. One important consideration is the $b a$ sis. So far we have assumed a monomial basis $1, x, x^{2}, \ldots$, and the arithmetic algorithms of the previous section work more or less the same over any basis. But interpolating into a different basis is more subtle. Sparse interpolation in Pockhammer, Chebyshev, and shifted power bases has been considered by [3, 35, 38, 50, 67].

Another interesting direction has been the development of more robust sparse interpolation algorithms, which can tolerate numerical noise in the evaluations, or completely-erroneous outliers, at the cost of performing more evaluations than in the exact setting $[3,15,18,59]$.

An even more difficult problem is sparse rational function interpolation, which is the same as sparse polynomial interpolation except that the unknown $f$ is a fraction of two sparse multivariate polynomials. Interestingly, [62] showed that the sparsest rational function is not always reduced; see also [20, 63, 64].

\section{FACTORIZATION}

The development of efficient algorithms to factor dense polynomials has been widely celebrated [33, 43, 54]. Most notably for our current purposes, since the 1980s it has been possible to factor polynomials over $\mathbb{Z}[x]$ in polynomial-time. Ignoring the thorny issues with multivariate polynomials and finite fields for now, we ask the same question for sparse polynomials over $\mathbb{Z}[x]$.

This question is already addressed in some other surveys such as $[22,26,54]$. Because of this, and since this is the area in which the speaker has the least expertise, we give only a very cursory overview of the accomplishments and challenges here.

\subsection{Impossibility results}

Plaisted [76] showed that the problem of determining whether two sparse polynomials are relatively prime is NP-complete, via a reduction from 3-SAT. This means that even computing the gcd of two supersparse polynomials is (seemingly) intractable. However, as highlighted by [22], it is important to emphasize that the reduction only uses cyclotomic polynomials; hence there is a possibility that by excluding such polynomials more progress is possible. 
Another impossibility is complete factorization, as illustrated by the (cyclotomic) example $x^{D}-1$, which has an exponentially-large dense factor.

The best we can hope for is perhaps the following:

Open Problem 7. Suppose $f \in \mathbb{Z}[x]$ is a $t$-sparse polynomial with at least one sparse factor $g \in \mathbb{Z}[x]$ such that $g$ has at most $s$ nonzero terms. In polynomial-time in $t, s, \log \mathrm{H}(f)$, and $\log \operatorname{deg} f$, find any $s$-sparse factor of $f$.

\subsection{Low-degree factors}

One case in which supersparse polynomial factorization is possible is when the factors are dense and have small degree. The results in this category generally depend on gap lemmas, whose statements are of the following form: If $f \in \mathrm{F}[x]$ can be written as $f=f_{0}+f_{1}$. $x^{k}$, where the "gap" $\left(k-\operatorname{deg} f_{0}\right)$ is large, then every non-cyclotomic factor of $f$ is a factor of both $f_{0}$ and $f_{1}$.

The actual gap lemmas are a bit more technical in specifying the gap and some other conditions, but what they tell us is that finding low-degree factors of a high-degree, sparse polynomial, can be reduced to finding factors of some dense sub-polynomial(s) of $f$ and then checking divisibility. (Recall from Section 2.3 that sparse divisibility testing is tractable when the divisor has low degree.)

This technique has been applied to degree- 1 factors in $\mathbb{Z}[x]$ [19], then to small degree over $\mathbb{Q}[x]$ [68], degree-2 in $\mathbb{Q}[x, y]$ [55], and finally small degree over $\mathbb{Q}\left[x_{1}, \ldots, x_{n}\right][17,39,56]$.

\subsection{High-degree factors}

Finding high-degree sparse factors remains a challenge in almost all cases. Very recent work by [1] proves that essentially all bivariate high-degree factors of a bivariate rational polynomial must be sparse, which provides some new hope that this problem is tractable.

Otherwise, the only high-degree sparse factorizations that can be computed efficiently are perfect roots of the form $f=g^{k}$ for some $k \in \mathbb{N}$. As shown by $[34,36]$, such factors $g$ can be computed when they exist and are sparse, and the power $k$ can be computed unconditionally, in polynomial-time in the sparse size of $f$. Interestingly, it can be proven that the power $k$ must be relatively small whenever $f$ is sparse; conversely, a high power of any polynomial is necessarily dense.

\section{ACKNOWLEDGMENTS}

This work was performed while the author was graciously hosted by the Laboratoire Jean Kuntzmann at the Université Grenoble Alpes.

This work was supported in part by the National Science Foundation under grants 1319994 (https://www.nsf.gov/awardsearch/ showAward?AWD_ID=1319994) and 1618269 (https://www.nsf.gov/ awardsearch/showAward?AWD_ID=1618269).

\section{REFERENCES}

[1] F. Amoroso and M. Sombra. Factorization of bivariate sparse polynomials. online, 2017. URL https://arxiv.org/abs/1710.11479.

[2] A. Arnold. Sparse Polynomial Interpolation and Testing. PhD thesis, University of Waterloo, 2016. URL http://hdl.handle.net/10012/10307.

[3] A. Arnold and E. L. Kaltofen. Error-correcting sparse interpolation in the chebyshev basis. ISSAC '15, pages 21-28. ACM, 2015. doi:10.1145/2755996.2756652.

[4] A. Arnold and D. S. Roche. Multivariate sparse interpolation using randomized Kronecker substitutions. ISSAC '14, pages 35-42. ACM, 2014. doi:10.1145/2608628.2608674
[5] A. Arnold and D. S. Roche. Output-sensitive algorithms for sumset and sparse polynomial multiplication. ISSAC '15, pages 29-36. ACM, 2015. doi:10.1145/2755996.2756653.

[6] A. Arnold, M. Giesbrecht, and D. S. Roche. Faster sparse interpolation of straightline programs. In V. P. Gerdt, W. Koepf, E. W. Mayr, and E. V. Vorozhtsov, editors, Proc. Computer Algebra in Scientific Computing (CASC 2013), volume 8136 of Lecture Notes in Computer Science, pages 61-74. Springer, September 2013. doi:10.1007/978-3-319-02297-0 5.

[7] A. Arnold, M. Giesbrecht, and D. S. Roche. Sparse interpolation over finite fields via low-order roots of unity. ISSAC '14, pages 27-34. ACM, 2014. doi:10.1145/2608628.2608671.

[8] A. Arnold, M. Giesbrecht, and D. S. Roche. Faster sparse multivariate polynomial interpolation of straight-line programs. Journal of Symbolic Computation, 2015. doi:10.1016/j.jsc.2015.11.005.

[9] M. Ben-Or and P. Tiwari. A deterministic algorithm for sparse multivariate polynomial interpolation. STOC '88, pages 301-309. ACM, 1988. doi:10.1145/62212.62241.

[10] F. Biscani. Parallel sparse polynomial multiplication on modern hardware architectures. ISSAC '12, 2012.

[11] R. E. Blahut. A universal reed-solomon decoder. IBM fournal of Research and Development, 28(2):150-158, March 1984. doi:10.1147/rd.282.0150.

[12] A. Borodin and P. Tiwari. On the decidability of sparse univariate polynomial interpolation. Computational Complexity, 1:67-90, 1991. doi:10.1007/BF01200058.

[13] A. Bostan, G. Lecerf, and E. Schost. Tellegen's principle into practice. ISSAC '03, pages 37-44. ACM, 2003. doi:10.1145/860854.860870.

[14] A. Bostan, F. Chyzak, M. Giusti, R. Lebreton, G. Lecerf, B. Salvy, and E. Schost. Algorithmes Efficaces en Calcul Formel. 1.0 edition, Aug. 2017.

[15] B. Boyer, M. T. Comer, and E. L. Kaltofen. Sparse polynomial interpolation by variable shift in the presence of noise and outliers in the evaluations. In Electr. Proc. Tenth Asian Symposium on Computer Mathematics (ASCM 2012), 2012.

[16] D. G. Cantor and E. Kaltofen. On fast multiplication of polynomials over arbitrary algebras. Acta Informatica, 28:693-701, 1991. doi:10.1007/BF01178683.

[17] A. Chattopadhyay, B. Grenet, P. Koiran, N. Portier, and Y. Strozecki. Computing the multilinear factors of lacunary polynomials without heights. Manuscript (submitted), 2013. URL https://arxiv.org/abs/1311.5694.

[18] M. T. Comer, E. L. Kaltofen, and C. Pernet. Sparse polynomial interpolation and Berlekamp/Massey algorithms that correct outlier errors in input values. ISSAC '12, pages 138-145. ACM, 2012. doi:10.1145/2442829.2442852.

[19] F. Cucker, P. Koiran, and S. Smale. A polynomial time algorithm for Diophantine equations in one variable. F. Symbolic Comput., 27(1):21-29, 1999. doi:10.1006/jsco.1998.0242.

[20] A. Cuyt and W. Lee. Sparse interpolation of multivariate rational functions. Theoretical Computer Science, 412(16):1445 - 1456, 2011. doi:10.1016/j.tcs.2010.11.050.

[21] A. Cuyt, W. shin Lee, and X. Wang. On tensor decomposition, sparse interpolation and Padé approximation. Faen journal on approximation, 8(1):33-58, 2016.

[22] J. H. Davenport and J. Carette. The sparsity challenges. In Symbolic and Numeric Algorithms for Scientific Computing (SYNASC), 2009 11th International Symposium on, pages $3-7$, Sept. 2009. doi:10.1109/SYNASC.2009.62.

[23] R. Fateman. Comparing the speed of programs for sparse polynomial multiplication. SIGSAM Bull., 37(1):4-15, March 2003. doi:10.1145/844076.844080.

[24] R. Fateman. Draft: What's it worth to write a short program for polynomial multiplication? Online, Dec. 2008. URL http://www.cs.berkeley.edu/ fateman/ papers/shortprog.pdf.

[25] R. J. Fateman. Endpaper: Frpoly: A benchmark revisited. LISP and Symbolic Computation, 4(2):155-164, Apr 1991. doi:10.1007/BF01813018.

[26] M. A. Forbes and A. Shpilka. Complexity theory column 88: Challenges in polynomial factorization. SIGACT News, 46(4):32-49, Dec. 2015. doi: $10.1145 / 2852040.2852051$.

[27] M. Fürer. Faster integer multiplication. STOC '07, pages 57-66. ACM, 2007. doi:10.1145/1250790.1250800.

[28] S. Garg and É. Schost. Interpolation of polynomials given by straightline programs. Theoretical Computer Science, 410(27-29):2659-2662, 2009. doi:10.1016/j.tcs.2009.03.030.

[29] M. Gastineau and J. Laskar. Development of TRIP: Fast sparse multivariate polynomial multiplication using burst tries. In V. Alexandrov, G. van Albada, P. Sloot, and J. Dongarra, editors, Computational Science - ICCS 2006, volume 3992 of Lecture Notes in Computer Science, pages 446-453. Springer Berlin Heidelberg, 2006. doi:10.1007/11758525_60.

[30] M. Gastineau and J. Laskar. Highly scalable multiplication for distributed sparse multivariate polynomials on many-core systems. In V. P. Gerdt, W. Koepf, E. W. Mayr, and E. V. Vorozhtsov, editors, Computer Algebra in Scientific Computing, pages 100-115, Cham, 2013. Springer International Publishing. doi:10.1007/9783-319-02297-0 8

[31] M. Gastineau and J. Laskar. Parallel sparse multivariate polynomial division. PASCO '15, pages 25-33. ACM, 2015. doi:10.1145/2790282.2790285.

[32] J. von zur Gathen and J. Gerhard. Modern Computer Algebra. Cambridge University Press, Cambridge, second edition, 2003. 
[33] J. von zur Gathen and D. Panario. Factoring polynomials over finite fields: A survey. Fournal of Symbolic Computation, 31(1-2):3 - 17, 2001. doi:10.1006/jsco.1999.1002.

[34] M. Giesbrecht and D. S. Roche. On lacunary polynomial perfect powers. ISSAC '08, pages 103-110. ACM, 2008. doi:10.1145/1390768.1390785.

[35] M. Giesbrecht and D. S. Roche. Interpolation of shifted-lacunary polynomials Computational Complexity, 19:333-354, 2010. doi:10.1007/s00037-010-0294-0.

[36] M. Giesbrecht and D. S. Roche. Detecting lacunary perfect powers and com puting their roots. Fournal of Symbolic Computation, 46(11):1242-1259, 2011. doi:10.1016/j.jsc.2011.08.006.

[37] M. Giesbrecht and D. S. Roche. Diversification improves interpolation. ISSAC '11, pages 123-130. ACM, 2011. doi:10.1145/1993886.1993909.

[38] M. Giesbrecht, E. Kaltofen, and W. Lee. Algorithms for computing sparsest shifts of polynomials in power, chebyshev, and pochhammer bases. Fournal of Symbolic Computation, 36(3-4):401 - 424, 2003. doi:10.1016/S0747-7171(03)00087-7. ISSAC 2002

[39] B. Grenet. Bounded-degree factors of lacunary multivariate polynomials. fournal of Symbolic Computation, 75:171-192, 2016. doi:10.1016/j.jsc.2015.11.013. Special issue on the conference ISSAC 2014: Symbolic computation and computer algebra.

[40] B. Grenet, J. van der Hoeven, and G. Lecerf. Randomized root finding over finite FFT-fields using tangent Graeffe transforms. In Proc. 40th International Symposium on Symbolic and Algebraic Computation, ISSAC '15, page to appear, 2015.

[41] D. Y. Grigoriev and M. Karpinski. The matching problem for bipartite graphs with polynomially bounded permanents is in NC. In Foundations of Computer Science, 1987., 28th Annual Symposium on, pages 166-172, Oct. 1987. doi:10.1109/SFCS.1987.56

[42] D. Y. Grigoriev, M. Karpinski, and M. F. Singer. Fast parallel algorithms for sparse multivariate polynomial interpolation over finite fields. SIAM fournal on Computing, 19(6):1059-1063, 1990. doi:10.1137/0219073.

[43] W. Hart, M. van Hoeij, and A. Novocin. Practical polynomial factoring in polynomial time. ISSAC '11, pages 163-170. ACM, 2011. doi:10.1145/1993886.1993914.

[44] D. Harvey, J. van der Hoeven, and G. Lecerf. Faster polynomial multiplication over finite fields. F. ACM, 63(6):52:1-52:23, Jan. 2017. doi:10.1145/3005344.

[45] J. van der Hoeven and G. Lecerf. On the complexity of multivariate block wise polynomial multiplication. In Proc. ISSAC 2012, pages 211-218, 2012. doi:10.1145/2442829.2442861.

[46] J. van der Hoeven and G. Lecerf. On the bit-complexity of sparse polynomial and series multiplication. Journal of Symbolic Computation, 50:227-0254, 2013 doi:10.1016/j.jsc.2012.06.004.

[47] J. van der Hoeven and G. Lecerf. Sparse polynomial interpolation in practice. ACM Commun. Comput. Algebra, 48(3/4):187-191, Feb. 2015 doi:10.1145/2733693.2733721.

[48] M.-D. A. Huang and A. J. Rao. Interpolation of sparse multivariate polynomials over large finite fields with applications. Fournal of Algorithms, 33(2):204-228, 1999. doi:10.1006/jagm.1999.1045.

[49] Q. Huang and X. Gao. Faster deterministic sparse interpolation algorithms for straight-line program multivariate polynomials. CoRR, abs/1709.08979, 2017. URL http://arxiv.org/abs/1709.08979.

[50] E. Imamoglu, E. L. Kaltofen, and Z. Yang. Sparse polynomial interpolation with arbitrary orthogonal polynomial bases. In Proc. ISSAC'18, 2018.

[51] S. M. M. Javadi and M. Monagan. Parallel sparse polynomial interpolation over finite fields. PASCO '10, pages 160-168. ACM, 2010. doi:10.1145/1837210.1837233.

[52] S. C. Johnson. Sparse polynomial arithmetic. SIGSAM Bull., 8:63-71, August 1974 doi:10.1145/1086837.1086847.

[53] E. Kaltofen. Notes on polynomial and rational function interpolation. Unpublished manuscript, 1988 .

[54] E. Kaltofen. Polynomial factorization: A success story. ISSAC '03, pages 3-4. ACM, 2003. doi: $10.1145 / 860854.860857$

[55] E. Kaltofen and P. Koiran. On the complexity of factoring bivariate supersparse (lacunary) polynomials. ISSAC '05, pages 208-215. ACM, 2005. doi:10.1145/1073884.1073914.

[56] E. Kaltofen and P. Koiran. Finding small degree factors of multivariate supersparse (lacunary) polynomials over algebraic number fields. ISSAC '06, pages 162-168. ACM, 2006. doi:10.1145/1145768.1145798.

[57] E. Kaltofen and W. Lee. Early termination in sparse interpolation algorithms. Journal of Symbolic Computation, 36(3-4):365-400, 2003. doi:10.1016/S07477171(03)00088-9. ISSAC 2002.

[58] E. Kaltofen and L. Yagati. Improved sparse multivariate polynomial interpolation algorithms. In P. Gianni, editor, Symbolic and Algebraic Computation, volume 358 of Lecture Notes in Computer Science, pages 467-474. Springer Berlin / Heidelberg, 1989. doi: $10.1007 / 3-540-51084-2 \_44$

[59] E. Kaltofen and Z. Yang. On exact and approximate interpolation of sparse rational functions. ISSAC '07, pages 203-210. ACM, 2007. doi:10.1145/1277548.1277577.

[60] E. Kaltofen, Y. N. Lakshman, and J.-M. Wiley. Modular rational sparse multivariate polynomial interpolation. ISSAC '90, pages 135-139. ACM, 1990. doi:10.1145/96877.96912.
[61] E. L. Kaltofen. Fifteen years after DSC and WLSS2: What parallel computations I do today [invited lecture at PASCO 2010]. PASCO '10, pages 10-17. ACM, 2010. doi:10.1145/1837210.1837213.

[62] E. L. Kaltofen and M. Nehring. Supersparse black box rational function interpolation. ISSAC '11, pages 177-186. ACM, 2011. doi:10.1145/1993886.1993916.

[63] E. L. Kaltofen and Z. Yang. Sparse multivariate function recovery from values with noise and outlier errors. ISSAC '13, pages 219-226. ACM, 2013. doi: $10.1145 / 2465506.2465524$.

[64] E. L. Kaltofen, C. Pernet, A. Storjohann, and C. Waddell. Early termination in parametric linear system solving and rational function vector recovery with error correction. ISSAC '17, pages 237-244. ACM, 2017. doi:10.1145/3087604.3087645.

[65] M. Khochtali, D. S. Roche, and X. Tian. Parallel sparse interpolation using small primes. PASCO '15, pages 70-77. ACM, 2015. doi:10.1145/2790282.2790290.

[66] L. Kronecker. Grundzüge einer arithmetischen Theorie der algebraischen Grössen. Journal für die reine und angewandte Mathematik, 92:1-122, 1882

[67] Y. N. Lakshman and B. D. Saunders. Sparse polynomial interpolation in nonstandard bases. SIAM fournal on Computing, 24(2):387-397, 1995. doi:10.1137/S0097539792237784.

[68] H. W. Lenstra, Jr. Finding small degree factors of lacunary polynomials. In Number theory in progress, Vol. 1 (Zakopane-Kościelisko, 1997), pages 267-276. de Gruyter, Berlin, 1999.

[69] M. Monagan and R. Pearce. Polynomial division using dynamic arrays, heaps, and packed exponent vectors. In V. Ganzha, E. Mayr, and E. Vorozhtsov, editors, Computer Algebra in Scientific Computing, volume 4770 of Lecture Notes in Computer Science, pages 295-315. Springer Berlin / Heidelberg, 2007. doi:10.1007/978-3540-75187-8_23.

[70] M. Monagan and R. Pearce. Parallel sparse polynomial multiplication using heaps. ISSAC '09, pages 263-270. ACM, 2009. doi:10.1145/1576702.1576739.

[71] M. Monagan and R. Pearce. Sparse polynomial division using a heap. Journal of Symbolic Computation, In Press, Corrected Proof, 2010. doi:10.1016/j.jsc.2010.08.014

[72] M. Monagan and R. Pearce. Sparse polynomial multiplication and division in maple 14. ACM Commun. Comput. Algebra, 44(3/4):205-209, Jan. 2011. doi:10.1145/1940475.1940521.

[73] M. Monagan and R. Pearce. POLY: A new polynomial data structure for maple 17. ACM Commun. Comput. Algebra, 46(3/4):164-167, Jan. 2013. doi:10.1145/2429135.2429173.

[74] M. Monagan and R. Pearce. The design of Maple's sum-of-products and POLY data structures for representing mathematical objects. ACM Commun. Comput. Algebra, 48(3/4):166-186, Feb. 2015. doi:10.1145/2733693.2733720.

[75] B. Mourrain. Fast algorithm for border bases of artinian gorenstein algebras. ISSAC '17, pages 333-340. ACM, 2017. doi:10.1145/3087604.3087632.

[76] D. A. Plaisted. New NP-hard and NP-complete polynomial and integer divisibility problems. Theoret. Comput. Sci., 31(1-2):125-138, 1984. doi:10.1016/03043975(84)90130-0.

[77] D. A. Popescu and R. T. Garcia. Multivariate polynomial multiplication on gpu. Procedia Computer Science, 80:154 - 165, 2016. doi:10.1016/j.procs.2016.05.306. International Conference on Computational Science 2016, ICCS 2016, 6-8 June 2016, San Diego, California, USA.

[78] B. d. Prony. Essai expérimental et analytique sur les lois de la Dilatabilité des fluides élastique et sur celles de la Force expansive de la vapeur de lâĂŹeau et de la vapeur de lâĂŹalkool, à différentes températures. F. de lâĂŹÉcole Polytechnique, 1:24-76, 1795 .

[79] D. S. Roche. Adaptive polynomial multiplication. In Proc. Milestones in Computer Algebra (MICA), pages 65-72, 2008.

[80] D. S. Roche. Chunky and equal-spaced polynomial multiplication. Fournal of Symbolic Computation, 46(7):791-806, July 2011. doi:10.1016/j.jsc.2010.08.013.

[81] H. Schönemann. Singular in a framework for polynomial computations. In M. Joswig and N. Takayama, editors, Algebra, Geometry and Software Systems, pages 163-176, Berlin, Heidelberg, 2003. Springer Berlin Heidelberg. doi:10.1007/978-3-662-05148-1 9 .

[82] J. Solomon. Numerical Algorithms. AK Peters/CRC Press, 2015.

[83] A. Steel. Multivariate polynomial rings. In The Magma Handbook. Computational Algebra Group, University of Sydney, 2018. URL http://magma.maths.usyd.edu. $\mathrm{au} / \mathrm{magma} / \mathrm{handbook} / \mathrm{text} / 223 \# 1924$

[84] W. Stein and T. Sage Development Team. Polynomial rings. In Sage Reference Manual. URL https://doc.sagemath.org/html/en/reference/polynomial_rings. v8.2.

[85] T. Yan. The geobucket data structure for polynomials. Journal of Symbolic Computation, 25(3):285-293, 1998. doi:10.1006/jsco.1997.0176.

[86] R. Zippel. Probabilistic algorithms for sparse polynomials. In E. Ng, editor, Symbolic and Algebraic Computation, volume 72 of Lecture Notes in Computer Science, pages 216-226. Springer Berlin / Heidelberg, 1979. doi:10.1007/3-54009519-5_73.

[87] R. Zippel. Interpolating polynomials from their values. Fournal of Symbolic Computation, 9(3):375-403, 1990. doi:10.1016/S0747-7171(08)80018-1. Computational algebraic complexity editorial. 